\title{
TOURS DE MAINS
}

Comme tant d'autres, la « main invisible " m'intrigue. Mais à des titres initiaux peut-être un peu différents.

Mon premier, comme il est dit dans les charades, est justement à cause de tous ces autres et de l'importance qu'ils ont conférée à l'expression, malgré l'usage discret que Smith en fait lui-même. Logée dans les plis et replis de la IV $V^{\mathcal{C}}$ Partie de La Théorie des sentiments moraux et du Livre IV de La Richesse des nations ${ }^{1}$, l'expression aurait tout aussi bien pu mener, des siècles durant, l'existence modeste d'un procédé rhétorique.

Mon deuxième est que l'alliance des termes, qui composent l'expression, est finalement curieuse.

Mon troisième, enfin, est que Smith donne lui-même la formule comme l'exemple type de la manière dont fonctionnait la pensée préscientifique $^{2}$. Dans ces conditions, à quoi peut bien correspondre qu'il en fasse lui aussi usage, même comme simple image? En devenant «une main invisible ", en devenant anonyme, «la main invisible de Jupiter" gagnerait-elle en rigueur ce qu'elle perd en ostentation?

1. On trouvera aux annexes I et II (infra, p. 199-201), la traduction des deux fragments où l'expression apparaît. Les abreviations TMS et $W N$ sont utilisées pour désigner respectivement chacun des deux ouvrages considérés. Les références dont ces abréviations sont suivies, sont celles adoptées par l'édition que l'Université de Glasgow a consacrée aux cuvres et à la correspondance d'Adam Smith. Les chiffres entre parenthèses indiquent les pages des volumes considérés. Se reporter à la Bibliographie p. 198-199.

2. On trouvera à l'annexe IV (infra, p. 202) l'extrait de Histoire de l'astronomie qui manifeste ce point de vue. L'abréviation $H A$ est utilisée pour désigner cet essai. Les références sont établies comme précédemment.

Revue de synthèse: IV $\mathrm{S} . \mathrm{N}^{\circ} 2$, avril-juin 1989. 


\section{D'UNE PHYSIOCRATIE À UNE COSMOPOLICE}

L'alliance des termes est vraiment curieuse.

Je ne crois pas céder complètement à la subjectivité en pensant que parler de « main » c'est suggérer plus qu'une présence, une intervention; et que qualifier cette main d' "invisible » c'est désigner l'intervention qu'elle suggère comme occulte. Si l'on m'accorde ce point de départ, surgit alors la question de savoir comment une telle intervention peut se repérer, ou même se pressentir.

$\mathrm{Au}$ cours du festin que le roi Balthazar donna pour ses seigneurs, non seulement la main qui soudainement apparut fut d'un réalisme tel qu'elle suscita l'effroi, mais l'inscription qu'elle laissa objectiva sans doute possible sa manifestation. Les Livres prophétiques en parlent ainsi :

« Soudain apparurent des doigts de main humaine qui se mirent à écrire derrière le lampadaire, sur le plâtre du mur du palais royal, et le roi vit la paume de la main ${ }^{3}$ qui écrivait $"{ }^{4}$.

Rien d'aussi effroyable dans l'œuvre d'Adam Smith. Il n'y a de main que le concept.

Telle que l'évoque Adam Smith, la «main invisible » pourrait davantage être rapprochée de "l'homme invisible ", qui fait l'objet du roman de H.G. Wells. De même que l'homme invisible n'avait pas pensé qu'aussi invisible fût-il son corps existait, de même, aussi réduite à un signifiant qu'elle soit, la « main invisible » présente quand même deux particularités.

La première est de ne pas s'exercer sur tous les individus, mais seulement sur certains d'entre eux : les "riches" (propriétaires fonciers $^{5}$, maîtres seigneuriaux) dans La Théorie des sentiments moraux; les « individus qui emploient leur capital » (éleveurs, manufacturiers,

3. Les traductions des récits archaïques comportent parfois des détails bizarres. Mais, à laquelle se fier?

4. Cf. Daniel, 5, 5. On sait qu'on fit appel à Daniel pour résoudre l'énigme de l'inscription tracée et qu'il la reconnut pour être Mené, Mené, Teqel et Parsîn, soit dans l'interprétation qu'il en fit : «Mené : Dieu a mesuré ton royaume et l'a livré; Teqel: tu as été pesé dans la balance et ton poids se trouve en défaut; Parsîn : ton royaume a été divisé et donné aux Mèdes et aux Perses" (ibid., 5, 25-29).

5. Littéralement, " seigneurs terriens". 
marchands) dans La Richesse des nations. Et puisque F. A. Hayek a cru bon à son sujet d'opposer taxis et kosmos $^{6}$, on peut déjà remarquer que la " main invisible ", en agissant de manière sélective, " classe ».

La deuxième particularité concerne, après ses créatures, son action. La main est invisible, non pas parce qu'elle est matériellement inapparente ou qu'elle opère sans laisser de trace, mais tout simplement parce qu'elle ne fait rien. Les « riches", sous son empire, ne cessent pas de céder à "leur rapacité et leur égoïsme naturels " et d'assouvir leurs « vains et insatiables désirs propres " (Théorie des sentiments moraux, cf. Annexe I, p. 199). Les "individus qui emploient leur capital ", quant à eux, ne cessent pas plus d'agir sans relâche pour leur "propre sécurité » et leur " propre gain " (Richesse des nations, cf. Annexe II, p. 200). Un vertige saisit alors le lecteur : si l'invisibilité de la main tient à son inaction, cette invisibilité n'est-elle pas purement et simplement une inexistence?

Mais il en est ici comme de tous les vertiges. La tentation du néant est l'effet de son amplification démesurée. L'inaction par laquelle se caractérise la "main invisible " n'est pas sans raison. Si la "main invisible" reste inerte, c'est qu'elle "sait ». Elle sait quelque chose que ne savent pas ses créatures d'élection. Elle sait le service public, que rend à leur insu leur acharnement à satisfaire leurs propres désirs vains ou leur gain propre, et également le bienfait pour tous qui résulte de cet insu.

Ainsi son inaction est motivée par ce qu'elle sait. Et ce qu'elle sait ne doit pas être su. Car, que les « riches ", ou les « individus qui emploient leur capital " viennent à avoir la conscience de ce dont ils sont inconscients, et c'en est fini du bien fait par leur aveuglement et, avec lui, du bénéfice que la société retire de leur conduite privée. Et puisque, que les mânes de Wittgenstein me pardonnent, "Wovon man nicht sprechen, darüber muß man schweigen ", puisque ce dont on ne peut parler, il faut le taire, comment une main pourrait-elle "se taire " autrement qu'en s'abstenant de geste? Son inaction est donc un mode d'action.

A ce point où simultanément l'abstention se révèle pour ce qu'elle est, une forme d'action, et l'invisibilité correspond à un aveuglement, la main, d'extérieure, se fait intérieure et parallèlement, de particulière, devient générale. Car l'aveuglement dont il s'agit est, quoiqu'on ne prête qu'aux riches, moins celui qui résulte de leur passion (Théorie des sentiments moraux) ou de la logique de leur fonds (Richesse des nations) que celui qui résulte d'une perception partagée des conduites, selon laquelle cellesci se résoudraient dans leur objet apparent et conscient. Suivant cette perception, la conduite des "riches" et des « individus qui emploient

6. F. A. HaYek, Droit, législation et liberté, Paris, P.U.F., 1980, t. 1, p. 43. 
leur capital " ne peut avoir pour terme exclusif que ce qui est à son motif : leur propre intérêt.

Or, si privée que soit leur conduite, elle ne peut faire qu'ils ne soient pas, comme tous les autres, des êtres soumis à la nature. La convoitise, qui dilate la pupille du « riche », ne parvient pas à augmenter à sa mesure immodérée la taille uniforme que la nature a impartie à l'estomac humain ${ }^{7}$. Et, à supposer qu'elle ne concerne pas que son estomac, les actions qu'elle suscite pour son contentement ne parviennent pas non plus à échapper à cette double détermination qui est à l'origine des sociétés humaines : d'une part, l'incapacité native dont les hommes sont frappés relativement à la satisfaction autonome de l'ensemble de leurs besoins divers et qui les fait dépendre les uns des autres; d'autre part, contrepartie de cette incapacité, la capacité à donner une forme juridique aux rapports de dépendance qui les relient, la capacité à les assujettir à des règles et à constituer ainsi, à la différence des autres espèces animales, leurs propres groupements en sociétés ${ }^{8}$. Êtres auto-insuffisants comparés aux êtres animaux, les hommes sont heureusement des êtres de raison, de langage et de droit et, comme tels, des êtres sociaux.

Aussi, conformément à la dépendance mutuelle dans laquelle, par nature, les hommes sont entre eux et qui fait qu'elles existent, les sociétés humaines sont-elles construites sur le principe que tout homme ne peut réaliser les buts qui lui sont propres que par le truchement obligé d'autrui. Tel est le sens unique, pour le donateur comme pour le donataire, du contenu do ut des des pactes nus : si je veux recevoir ce ne peut être qu'en donnant. Aussi les « riches " ne peuvent-ils assouvir leurs vains et insatiables désirs propres, et les « individus qui emploient leur capital » assurer la mise en valeur renouvelée de celui-ci, sans recourir à l'industrie d'autrui (Théorie des sentiments moraux) et sans contribuer par là à l'industrie générale (Richesse des nations). Ou encore, ils ne le peuvent sans rétrocéder pour partie ce «trop » qu'ils ont obtenu provi-

7. Smith fait valoir l'opinion non seulement dans La Théorie des sentiments moraux, mais aussi dans La Richesse des nations : "Le désir de nourriture est limité en tout homme par l'étroitesse de la capacité de l'estomac humain; mais le désir de commodités et d'ornements en fait de construction, d'habillement, d'équipage, et d'ameublement, semble n'avoir point de limite ou de frontière déterminée. Ceux, donc, qui ont le commandement sur plus de nourriture qu'ils ne peuvent eux-mêmes en consommer, sont toujours prêts à échanger le surplus, ou, ce qui revient au même, le prix de ce surplus, contre des contentements de cette autre sorte. Ce qui excède la satisfaction du désir limité est donné contre l'amusement de ces désirs qui ne peuvent pas être satisfaits, mais qui semblent être absolument sans limite. Les pauvres, pour obtenir de la nourriture, s'efforcent de contenter ces fantaisies des riches, et pour l'obtenir plus sûrement, rivalisent entre eux de bon marché et de perfection dans leur ouvrage ". Cf. $W N, I$, xi, c, 7, 1, p. 181 .

8. On trouvera à l'annexe III (infra, p. 201) le passage de La Richesse des nations auquel j'emprunte, ici et pour les arguments qui suivent. 
dentiellement en partage (Théorie des sentiments moraux), ni sans concourir à la mise en valeur du capital social et de son produit (Richesse des nations).

Sous cet angle, la « main invisible qui guide riches » et « individus qui emploient leur capital » n'est pas autre chose que la dépendance dans laquelle tous les hommes sont, par nature, entre eux et qu'ils ont enregistrée dans les sociétés qu'ils ont formées. Elle n'est donc pas autre chose que l'ordre de la nature, ou son double social, si inscrits au fond de leurs conduites que celles-ci leur échappent le moins au moment où elles semblent leur échapper le plus.

La «main invisible » est la prévalence de l'harmonie de la nature, et de la société qui la décalque, sur les hommes. En ce sens elle est bien kosmos, parce que, référant fondamentalement au "bon ordre » (sous les deux aspects de "bienséance » ou "discipline " et de "gouvernement »), kosmos n'a pas seulement le sens d' "ordre du monde», d' « univers », mais parce qu'il a aussi celui de " constitution » ; particulièrement en Crète, il désigne le " magistrat suprême ", dont la fonction est de maintenir le bon ordre, d'être le « régulateur». En ce sens, si la « main invisible » est kosmos, elle est aussi taxis. Car l'un peut ne pas aller sans l'autre. Taxis ne signifie pas seulement « rangement » militaire, mais aussi "rangement " administratif, donc " ordre politique " ou "constitution". A suivre Socrate d'ailleurs, les deux termes, loin de s'opposer, s'unissent pour désigner les composantes majeures qui font la qualité d'une maison comme d'un citoyen'.

Ainsi plutôt que cosmi-économique, qui fait par proximité songer à « cosmicomics » forgé par Italo Calvino, la « main invisible " est « cosmopolitique » et vient recadrer la poigne physiocratique qui, réduisant la nature à la terre, porte à l'absolu le système économique sans respecter l'équilibre des pouvoirs établis constitutifs du système politique.

\section{DU THÊÂTRE DE LA NATURE AU SPECTACLE DE SON SYSTÈME}

Si dans cette interprétation se conçoit mieux qu'Adam Smith ait figuré par une main l'emprise qu'exerce sur les conduites des hommes, fussent-

9. Cf. Platon, Gorgias, 504a : "L'ordre et l'harmonie [táxis kai kósmos] font donc la bonne qualité d'une maison, tandis qu'avec le désordre elle est sans valeur "; ibid., 504d : " Dans l'âme, l'ordre et l'harmonie s'appellent la discipline et la loi, qui font les bons citoyens et les honnêtes gens : et c'est cela qui constitue la justice et la sagesse. " Voir aussi ibid., 506c-507a. 
ils les plus puissants et les plus égoïstes, leur dépendance sociale, et qu'il ait rendu son caractère abstrait par l'invisibilité, reste encore un hic : la dénotation de cette emprise abstraite que débusque la raison par une représentation, dont Adam Smith nous dit lui-même qu'elle est le signe de la raison prise en défaut, de sa stupeur ${ }^{10}$.

Dans Histoire de l'astronomie ${ }^{11}$, Adam Smith caractérise la pensée philosophique, ou scientifique ${ }^{12}$, par opposition aux limbes de la pensée, ou pensée " primitive ». Selon lui, la première a cette particularité d'intégrer les irrégularités qui se produisent dans les repères réguliers du monde en reconstituant rationnellement le système d'ensemble. Elle se différencie ainsi de la seconde qui, absorbée par le régulier, est conduite à rejeter dans l'irrationnel tout ce qui le trouble et la trouble le plus apparemment et, sous l'empire des sentiments que cet irrationnel suscite, à imputer ces perturbations au dessein de fauteurs surnaturels. Tel est le sens de l'attribution par les Anciens à « l'invisible main de Jupiter ", chargée selon les cas de manifester son courroux ou sa bienveillance, des événements cosmiques qui les plongeaient dans la terreur ou, scellant leur élection, au contraire dans la vénération.

Dans les conditions créées par cette opposition, comment comprendre la dissociation qui, à confronter les différentes pièces du dossier ( $L a$ Théorie des sentiments moraux et La Richesse des nations d'un côté, Histoire de l'astronomie de l'autre), semble s'opérer dans l'esprit d'Adam Smith entre son propre recours à la « main invisible » et celui qu'y ferait la pensée primitive, archaïque ou sauvage? Car, après tout, la fonction qu'Adam Smith lui assigne est-elle si différente de celle que lui assignerait la mentalité primitive?

Ce qu'il y a de frappant dans l'analyse qu'Adam Smith fait de cette mentalité, et sur quoi il insiste, c'est que celle-ci borne l'intervention des divinités qu'elle invoque uniquement aux manifestations naturelles qui ont effet d'effroi ou, ce qui revient au même, de gratitude. Ces manifestations naturelles (comètes, éclipses, tonnerre, éclair, tempêtes, météores)

10. Cf. $H A$, III, 1, p. 48 : « Beaucoup de ces petites incohérences, qui dans le cours des choses rendent perplexes les philosophes, échappent à son attention [celle du sauvage]. Les irrégularités plus grandioses, dont il ne peut négliger la puissance, provoquent sa stupeur. "

11. Ce texte figure dans Essais sur des sujets philosophiques. Le titre complet en est : Les Principes qui guident et dirigent les enquêtes philosophiques; illustrés par l'histoire de l'astronomie. L'extrait relatif à la question considérée est donné à l'annexe IV, infra, p. 202.

12. Cf. $H A$, III, 3, p. 50 : «C'est donc l'étonnement, et non l'attente d'avantage tiré de ces découvertes, qui est le premier principe qui porte l'humanité à l'étude de la Philosophie, de cette science qui prétend mettre à découvert les liaisons cachées qui unissent les apparences diverses de la nature; $[\ldots]$ » 
sont toutes de celles qui échappent au contrôle des hommes, même si depuis elles n'échappent plus à leur connaissance. Or un incontrôlé gît aussi, latent, dans La Théorie des sentiments moraux et dans La Richesse des nations. Il est cette fois relatif aux hommes eux-mêmes. C'est même au moment précis où il est question de la « main invisible » qu'il existe le plus. Ou, en présentant les choses à l'envers, c'est au moment où Smith plaide en faveur de la conformité de la puissance publique aux pouvoirs établis ${ }^{13}$ (Théorie des sentiments moraux) ou de son non-engagement en matière économique (Richesse des nations) qu'il en appelle à la « main invisible ». Car que peut tirer l'opinion, dont Smith se montre si soucieux, de ce vide public, si ce n'est la crainte que les forts n'en profitent et que, loin de se réduire, les écarts existants ne se creusent davantage encore, et sans rémission?

Au regard de cette crainte et de ses ramifications archaïques, la « main invisible " pourrait servir à Adam Smith de la même façon qu'elle aurait servi aux Anciens. Elle servirait à conjurer la plus primitive des craintes, celle de l'incontrôlé, par l'appel au plus primitif des moyens, le surnaturel.

Mais à ce point de convergence s'aperçoit une différence. Si la « main invisible » peut avoir, maintenant, la même fonction que celle qu'elle aurait eu anciennement, elle ne la remplit plus de la même façon. Ses interventions ne sont plus associées, comme elles l'auraient été anciennement, aux troubles les plus éclatants de ce qui passe pour l'ordre. Elles sont bien plutôt données comme exprimant l'ordre caché toujours présent dans ce qui semble être le désordre. C'est qu'entre-temps la nature a changé d'apparence : de disjoints qu'ils semblaient ses phénomènes divers ont été reliés et dans leur liaison ont révélé un assemblage confondant. Ce qui frappe désormais, ce qui est « surnaturel », ce ne sont plus les événements magnifiques dont la nature est le théâtre : ses désordres, mais le spectacle qu'elle offre à la spéculation : son ordre. Le surnaturel ne peut plus être pensé à l'image des hommes qui n'agissent jamais que pour arrêter, contrarier et troubler le cours des choses. Il ne peut être référé qu'à un existant plus grand qu'eux-mêmes. Et cet existant ne peut

13. Cf. $T M S$, VI, ii. 2,17 , p. 234 : « Il [l'homme de système] semble imaginer qu'il peut disposer les différents membres d'une grande société, avec autant de facilité que la main dispose les pièces sur un échiquier. Il ne considère pas que sur l'échiquier les pièces n'ont aucun autre principe de mouvement que celui que leur imprime la main; mais que, dans le grand échiquier de la société humaine, chaque pièce particulière a un principe de mouvement qui lui est propre, tout à fait différent de celui que la législature pourrait choisir de lui imprimer. Si ces deux principes coïncident et agissent dans la même direction, le jeu de la société humaine se poursuivra facilement et harmonieusement, et selon toute probabilité, sera heureux et réussi. S'ils sont opposés ou différents, le jeu se poursuivra misérablement, et la société connaîtra en tout temps le degré le plus haut de désordre. " 
que s'associer à l'Auteur à l'image duquel, avant la Chute, les hommes ont été faits. Aux dieux et à leurs caprices humains succèdent Dieu et sa Providence.

Sous cet angle apparait alors l'ancienneté de l'idéalité qui fait écrire à Smith :

"Quand la Providence partagea la terre entre un petit nombre de maitres seigneuriaux, elle n'a ni oublié, ni abandonné ceux qui semblaient avoir été exclus du partage. Ces derniers aussi jouissent de leur part de tout ce qu'elle produit. Dans ce qui constitue le bonheur réel de la vie humaine, ils ne sont en rien inférieurs à ceux qui sembleraient tellement plus au-dessus d'eux. Quant au bien-être du corps et à la paix de l'esprit, tous les différents rangs de la vie sont presque au même niveau, et le mendiant qui se chauffe au soleil sur le bord de la route, possède cette sécurité pour laquelle les rois se battent $" 14$.

Plus d'un siècle avant, Bacon disposait de la même façon :

"L'Exemple de Dieu enseigne la vraie Leçon : Il envoie sa Pluie et fait briller son Soleil sur le Juste et l'Injuste; Mais il ne fait pas pleuvoir la Richesse ni briller l'Honneur et les Vertus 'également sur les Hommes. Les Bienfaits communs doivent être communiqués à tous, Mais les Bienfaits singuliers, avec choix $" 15$.

Si la " main invisible " peut être employée quoique celle " de Jupiter " ne puisse plus l'être, c'est d'abord que la science conclut à Dieu quoiqu'elle ne soit telle que d'avoir rejeté les dieux. Ou encore, c'est que la pensée scientifique ne considère pas qu'elle s'abolit comme telle lorsque, par exemple, elle s'élève des lois de la gravité à " la domination d'Un seul " ${ }^{16}$. La " main invisible » dont Smith s'autorise, bien qu'il tienne en s'aidant de Malebranche ${ }^{17}$ celle de Jupiter pour une rationalisation, appartient encore à cette conception de la science. Elle est comme l'écho affaibli par un siècle de distance du scolie général inséré par Isaac Newton dans les Philosophice Naturalis Principia Mathematica. Car en un siècle l'Être de Newton, déjà vide de tout corps et de toute forme

14. TMS, IV, i, 9, p. 183-184). Voir Annexe I, infra, p. 199-200.

15. Francis BACON, The Essays or Counsels, Civill and Morrall, London, Iohn Havilland, 1625.

16. Isaac NEwTon, Principia, III, Berkeley/Los Angeles/London, University of California Press, 1962, t. 2, p. 544.

17. Cf. $H A$, III, 1, p. 48 : « Mais nos passions, comme l'observe le Père Malbranche [sic], se justifient toutes, c'est-à-dire qu'elles nous suggèrent les opinions qui peuvent les justifier. " 
physique, s'est, s'il est possible, encore plus dépouillé : la " main invisible ", qui n'était plus « de Jupiter », est posée sans plus aucune sorte de référence. Elle est « une » main invisible.

On peut se demander si ce n'est pas à cette inscription théologique que la "main invisible " doit la fascination qu'elle exerce. Sa fortune ne tiendrait alors qu'au sort qu'on tâche de faire à un élément qui, depuis que la science ne pense plus dans l'espace théologique et ne se pense plus comme telle dans cet espace, contrarie l'existence de l'économie comme science, science à part et science à part entière. De là viendrait cette insistance à soutenir que la " main invisible » n'est pas autre chose que l'intuition des vertus d'ordre que présenterait spontanément un marché où jouerait parfaitement la concurrence libre.

Prendre cette option c'est, en fait, rabattre l'un sur l'autre les deux registres distincts qu'Adam Smith fait jouer : le système fondamental, ou le système des obligations par lesquelles les hommes s'imbriquent en corps social ; et son auxiliaire. Par l'un, il fait apparaître les effets-retours sur les conduites privées que provoque l'imbrication sociale et par là l'attraction que la société exerce sur elles. Par l'autre, il fait apparaître quel dispositif peut seul compléter ce système fondamental et le perpétuer en favorisant la réponse adaptée à toute modification survenant de manière externe. Au premier correspond la « main invisible », au second le marché concurrentiel, que Smith, à la différence de Quesnay, n'envisageait pas systématiquement parfait ${ }^{18}$.

18. Cf. $W N, I V$, ix, 28,2, p. 673-674 : « Quelques médecins spéculatifs semblent avoir imaginé que la santé du corps humain ne pourrait être préservée que par un certain régime précis de diète et d'exercice, dont toute violation fût-elle la plus minime entraînerait nécessairement un certain degré de maladie ou de désordre proportionné au degré de la violation. L'expérience, cependant, semblerait montrer que le corps humain conserve fréquemment, du moins selon toute apparence, l'état le plus parfait de santé sous une grande diversité de régimes différents; même sous certains qui sont généralement tenus pour être loin d'être parfaitement salutaires. [...] M. Quesnai [sic], qui était lui-même un médecin, et un médecin très spéculatif, semble avoir conçu une notion du même genre concernant le corps politique, et avoir imaginé qu'il ne serait florissant et ne prospérerait que sous un certain régime précis, le régime strict de parfaite liberté et de parfaite justice. [...] Si une nation ne pouvait prospérer sans jouir de la liberté parfaite et de la justice parfaite, aucune nation au monde n'eût jamais pu prospérer. Dans le corps politique, cependant, la sagesse de la nature heureusement a amplement prévu de quoi remédier à bien des mauvais effets de la folie et de l'injustice de l'homme; de la même manière qu'elle l'a fait dans le corps naturel pour remédier à ceux de son indolence et de son intempérance. » 
En assimilant celui-ci à la Providence, il n'est pas sûr que l'économie prenne rang de science, au sens où nous l'entendons; et, en considérant l'auxiliaire comme le fondamental, qu'elle établisse son hégémonie. A bénéficier d'attributs qui ne sont pas les siens, mais ceux de la société, le marché perd plus qu'il ne gagne. Sa théorie reste toujours confrontée au mystère par lequel il parviendrait à réaliser ce qui procède de la constitution des sociétés et du dessein qu'elle traduit. Reposant sur une équivoque, la théorie concurrentielle véhicule et entretient une théologie du marché. Ce n'est ni troublant, ni accablant. C'est à rétablir dans nos mémoires athées.

Paulette TAIEB, Université Paris $I$.

\section{BIBLIOGRAPHIE}

Adam SMrth, The Theory of Moral Sentiments, ed. by D.D. RAPHAEL \& A.C. MACFIE, Oxford, Clarendon Press, 1979.

Adam SMIrH, An Inquiny into the Nature and Causes of the Wealth of Nations, eds R. H. Campbell, A.S. Sxinner \& W.B. TodD, Oxford, Clarendon Press, 1976.

Adam Smrth, Essays on Philosophical Subjects, ed. by W.P.D. WhightMan, J.C. BryCE \& I. S. Ross, Oxford, Clarendon Press, 1980.

Pierre Chantraine, Dictionnaire étymologique de la langue grecque, I-II, IV.1, Paris, Klincksieck, 1968.

H.G. LIDDELL, R. ScotT, A Greek-English Lexicon, Oxford, Clarendon Press, 1979.

Aristote, Politique, Paris, Les Belles Lettres, 1968, 3 t.

Bible.

Francis BACON, The Essays or Counsels, Civill and Morrall, London, Iohn Havilland, 1625.

Italo Calvino, Cosmicomics, Paris, Seuil, 1968.

F. A. HAYEK, Droit, législation et liberté, trad. de l'anglais par Raoul AudouIn, Paris, P.U.F., 1980.

Gottfried Wilhelm LeIBNIZ, Essais de théodicée, Paris, GF/Texte Intégral 209, 1969.

MAlebranche, De la recherche de la vérité, Paris, Vrin, 1946. 
Malebranche, Traité de la nature et de la grâce, Paris, Vrin, 1958.

Isaac Newion, Mathematical Principles of Natural Philosophy and his System of the World, Berkeley/Los Angeles/London, University of California Press, 1962.

Platon, Gorgias, Paris, Les Belles Lettres, 1974.

François QUESNAY, "Droit naturel », in François Quesnay et la physiocratie, Paris, I.N.E.D., 1958, t. 2.

Herbert George Wells, L'Homme invisible, Paris, Le Livre de Poche 709, 1986.

Ludwig WITTGENSTEIN, Tractatus logico-philosophicus, Paris, Gallimard, « Idées », 1972.

\section{ANNEXES : fragments d'Adam Smith \\ (sources complètes indiquées en Bibliographie, p. 198)}

« Et il est bien que la nature nous abuse de cette façon. C'est ce leurre qui suscite l'industrie de l'humanité et la tient en mouvement continuel. C'est ce qui la porta d'abord à cultiver le sol, édifier des maisons, fonder des cités et des républiques, et à inventer et améliorer tous les arts et les sciences, qui ennoblissent et embellissent la vie humaine; qui ont entièrement modifié la face entière du globe, ont changé les rudes forêts de la nature en plaines agréables et fertiles, et fait du vierge et stérile océan un fond nouveau de subsistance, et la grande voie de communication vers les différentes nations de la terre. La terre par ces travaux de l'humanité a été obligée de redoubler sa fertilité naturelle, et d'entretenir une plus grande multitude d'habitants. C'est inutilement, que l'orgueilleux et insensible propriétaire foncier contemple ses vastes champs, et sans une pensée pour les besoins de ses frères, qu'il consomme en imagination la totalité de la récolte qu'il y fait croître. Jamais ne fut plus complètement vérifié qu'à son égard le proverbe simple et vulgaire, selon lequel l'œil est plus gros que le ventre. Son estomac n'a pas une capacité proportionnée à l'immensité de ses désirs, et ne contiendra pas plus que celui du plus humble paysan. Le reste, il est obligé de le distribuer parmi ceux qui préparent, de la façon la plus délicieuse, $£$ peu que luimême utilise, parmi ceux qui aménagent le palais dans lequel ce peu doit être consommé, parmi ceux qui pourvoient et tiennent en ordre tous les différents colifichets et babioles, qui sont employés dans l'œconomie de faste; tous tirant ainsi de son luxe et de son caprice, cette part des nécessités de la vie, qu'ils attendraient en vain de son humanité ou de sa justice. Le produit du sol entretient en tout temps presque le nombre d'habitants qu'il est capable d'entretenir. Les riches choisissent seulement dans le tas ce qui est le plus précieux et le plus agréable. Ils ne consomment guère plus que les pauvres, et en dépit de leur rapacité et de leur égoïsme naturels, quoiqu'ils ne prétendent qu'à leur propre commodité, quoique la seule fin qu'ils visent à partir des travaux de tous les milliers de gens qu'ils emploient, soit le contentement de leurs vains et insatiables désirs propres, ils partagent avec les pauvres le produit de toutes leurs améliorations. Ils sont conduits par une main invisible à faire presque la même distribution des nécessités de la vie, que celle qui aurait été faite, si la terre avait été partagée 
en portions égales entre tous ses habitants, et ainsi sans en avoir l'intention, sans le savoir, ils font avancer l'intérêt de la société, et permettent la multiplication de l'espèce. Quand la Providence partagea la terre entre un petit nombre de maîtres seigneuriaux, elle n'a ni oublié, ni abandonné ceux qui semblaient avoir été exclus du partage. Ces derniers aussi jouissent de leur part de tout ce qu'elle produit. Dans ce qui constitue le bonheur réel de la vie humaine, ils ne sont en rien inférieurs à ceux qui sembleraient tellement plus au-dessus d'eux. Quant au bienêtre du corps et à la paix de l'esprit, tous les différents rangs de la vie sont presque au même niveau, et le mendiant qui se chauffe au soleil sur le bord de la route, possède cette sécurité pour laquelle les rois se battent " (TMS, IV, i, 9, p. 183184).

« En entravant, soit par des droits élevés, soit par des interdictions absolues, l'importation de pays étrangers de marchandises qui peuvent être produites à l'intérieur, on assure plus ou moins à l'industrie domestique employée à les produire le monopole du marché intérieur. [...]

Que ce monopole du marché intérieur donne fréquemment un grand encouragement à l'espèce déterminée d'industrie qui en jouit, et qu'il dirige fréquemment vers cet emploi une part à la fois du travail et du fonds de la société plus grande que celle qui s'y serait autrement dirigée, est indubitable. Mais qu'il tende à accroître l'industrie générale de la société, ou à lui donner la direction la plus avantageuse, n'est peut-être pas tout à fait si évident.

L'industrie générale de la société ne peut jamais dépasser ce que le capital de la société peut employer. De même que le nombre d'ouvriers qui peuvent être pourvus d'emploi par un individu donné doit avoir une certaine proportion avec son capital, de mème le nombre de ceux qui peuvent être continuellement employés par tous les membres d'une grande société, doit avoir une certaine proportion avec le capital de cette société, et ne peut jamais dépasser cette proportion. Il n'est pas de règlement de commerce qui puisse accroître la quantité d'industrie dans une société au-delà de ce que son capital peut entretenir. Un règlement de commerce ne peut qu'en dévier une partie dans une direction qu'elle n'aurait pas pu prendre autrement; et il n'est nullement certain que cette direction artificielle soit susceptible d'être plus avantageuse à la société que celle que l'industrie aurait prise d'elle-même.

Tout individu s'efforce continuellement de trouver l'emploi le plus avantageux à tout le capital qu'il peut commander. C'est son propre avantage, il est vrai, et non celui de la société qu'il a en vue. Mais la recherche de son propre avantage le conduit naturellement, ou plutôt nécessairement, à préférer précisément l'emploi qui est le plus avantageux à la société.

Premièrement, tout individu tâche d'employer son capital aussi près de lui qu'il peut, et par conséquent autant qu'il peut de l'employer au soutien de l'industrie domestique; pourvu qu'il puisse par là toujours obtenir les profits ordinaires du fonds, ou pas beaucoup moins que ceux-ci. [...]

Deuxièmement, tout individu qui emploie son capital au soutien de l'industrie domestique, tâche nécessairement de diriger cette industrie, de telle sorte que son produit soit de la plus grande valeur possible.

Le produit de l'industrie est ce qu'elle ajoute au sujet, ou aux matières, sur lesquels elle est employée. Suivant que la valeur de ce produit est grande ou 
petite, il en sera de mème des profits de l'employeur. Mais c'est seulement en vue du profit qu'un homme emploie un capital au soutien de l'industrie; et, donc, il tâchera toujours de l'employer au soutien de l'industrie dont le produit est susceptible d'être de la plus grande valeur, ou de s'échanger contre la plus grande quantité soit d'argent soit d'autres marchandises.

Mais le revenu annuel de toute société est toujours précisément égal à la valeur échangeable de la totalité du produit annuel de son industrie, ou plutôt est précisément la même chose que cette valeur échangeable. Comme tout individu, donc, tâche autant qu'il peut à la fois d'employer son capital au soutien de l'industrie domestique, et de diriger cette industrie de telle sorte que son produit puisse être de la plus grande valeur; tout individu travaille nécessairement à rendre le revenu annuel de la société aussi grand qu'il le peut. Généralement, il est vrai, ni il n'entend avancer l'intérêt public, ni il ne sait de combien il l'avance. En préférant le soutien de l'activité domestique à celui de l'activité étrangère, il ne vise que sa propre sécurité ; et en dirigeant cette activité d'une façon telle que son produit puisse être de la plus grande valeur, il ne vise que son propre gain, et il est en ceci, comme en beaucoup d'autres cas, conduit par une main invisible pour avancer une fin qui n'entrait point dans son intention. Et ce n'est pas toujours le pire pour la société qu'elle n'y entrât point. En poursuivant son propre intérêt il avance souvent celui de la société plus efficacement que s'il y visait réellement. Je n'ai jamais vu beaucoup de bien fait par ceux qui affectaient de commercer pour le bien public. Ce n'est pas, il est vrai, une affectation très courante parmi les marchands, et il suffit de très peu de mots pour les en dissuader " $(W N, I V$, ii, $1-10,1$, p. 452-456).

«Cette division du travail, d'où proviennent tant d'avantages, n'est originellement l'effet d'aucune sagesse humaine, qui prévoit et projette cette opulence générale à laquelle elle donne lieu. C'est la conséquence nécessaire, quoique très lente et graduelle d'une certaine propension de la nature humaine qui n'a pas pour fin une utilité aussi considérable; la propension à changer, troquer et échanger une chose contre une autre.

On n'examinera pas ici la question de savoir si cette propension est un des principes originels de la nature humaine, qui n'admet pas plus d'explication; ou si, comme il semble plus probable, elle est la conséquence nécessaire de la faculté de raison et de celle de la parole. Elle est commune à tous les hommes et ne se retrouve dans aucune autre race d'animaux, qui ne semble connaitre ni celle-ci ni aucune autre espèce de contrat. Deux lévriers qui forcent le même lièvre, ont parfois l'air d'agir en quelque sorte de concert. Chacun dirige la hase vers son compagnon, ou tâche de l'intercepter quand son compagnon la dirige vers lui. $\mathrm{Ce}$ n'est, cependant, pas là l'effet d'un contrat, mais du concours accidentel de leurs passions pour le même objet à un moment particulier. Personne n'a jamais vu un chien faire avec un autre chien un échange loyal et intentionnel d'un os contre un autre. Personne n'a jamais vu un animal par ses gestes et ses cris naturels signifier à l'autre, ceci est à moi, cela est à toi ; je suis prêt à donner ceci contre cela. Quand un animal désire obtenir quelque chose d'un homme ou d'un autre animal, il n'a pas d'autres moyens de persuasion que de gagner la faveur de ceux auxquels il demande service. Un chiot fait des caresses à sa mère, et un épagneul, quand il désire que son maître qui dîne le nourrisse, tâche par mille séductions d'attirer 
son attention. L'homme fait parfois usage des mêmes arts avec ses frères, et quand il n'a pas d'autres moyens de les engager à agir selon ses inclinations, il tâche par toute attention servile et flatteuse d'obtenir leur bon vouloir. Il n'a pas le temps, cependant, d'agir de la sorte en toute occasion. Dans la société civilisée il a à tout moment besoin de la coopération et de l'aide de grandes multitudes, tandis que sa vie toute entière est à peine suffisante pour gagner l'amitié d'un petit nombre de personnes. Dans presque toute autre race d'animaux chaque individu, quand il est parvenu à maturité, est entièrement indépendant, et dans son état naturel n'a besoin de l'aide d'aucune créature vivante. Mais l'homme a presque constamment besoin du secours de ses frères, et il est vain pour lui de ne l'attendre que de leur bienveillance. Il aura plus de chance de réussir, s'il peut intéresser leur amour de soi en sa faveur, et leur montrer qu'il est de leur avantage de faire pour lui ce qu'il leur demande. Quiconque offre à un autre un marché de quelque sorte que ce soit, propose de faire ceci. Donne-moi ce dont j'ai besoin, et tu auras ce dont tu as besoin, est le sens de toute offre de cette sorte; et c'est de cette façon que nous obtenons les uns des autres la plus grande partie des bons offices dont nous avons besoin. Ce n'est pas de la bienveillance du boucher, du brasseur, ou du boulanger, que nous attendons notre dîner, mais du souci qu'ils ont de leur propre intérêt. Nous ne nous adressons pas à leur humanité, mais à leur amour de soi, et nous ne leur parlons jamais de nos propres besoins, mais de leurs avantages. Personne sauf un mendiant ne choisit de dépendre principalement de la bienveillance de ses concitoyens. Même un mendiant n'en dépend pas entièrement. La charité des gens bien disposés lui fournit, il est vrai, la totalité de son fond de subsistance. Mais quoique ce principe le pourvoit à la longue de toutes les nécessités de la vie dont il a besoin, ni il ne le fait ni il ne peut le faire autant que le mendiant en a besoin. La plus grande partie de ses besoins ponctuels sont fournis de la même manière que ceux des autres gens, par traité, par troc, et par achat. Avec l'argent qu'un homme lui donne il achète de la nourriture. Les vieux habits qu'un autre lui octroye il les échange contre d'autres vieux habits qui lui conviennent mieux, ou contre le gîte, ou contre de la nourriture, ou contre de l'argent, avec lequel il peut acheter de la nourriture, des habits, ou le gite, selon le besoin qu'il en a " $(W N, \mathrm{I}, \mathrm{i}, 1-2$, p. 25-27).

« Mais un sauvage, dont les notions sont entièrement guidées par la nature sauvage et la passion, n'attend pas d'autre preuve qu'une chose est l'objet adéquat d'un sentiment quelconque, que le fait qu'elle le suscite. La révérence et la gratitude, que certaines des apparences de la nature lui inspirent, le convainquent qu'elles sont les objets adéquats de révérence et de gratitude, et qu'elles procèdent donc de certains êtres intelligents, qui trouvent du plaisir dans les expressions de ces sentiments. Pour lui, donc, tout objet de la nature, qui par sa beauté ou sa grandeur, son utilité ou sa nocivité, est assez considérable pour attirer son attention, et dont les opérations ne sont pas parfaitement régulières, est supposé agir sous la direction de quelque pouvoir invisible et doué d'intention. La mer s'apaise étale, ou se soulève tempétueuse, selon le bon plaisir de Neptune. La terre livre-t-elle une moisson foisonnante? La cause en est dans l'indulgence de Cérès. La vigne rend-elle une vendange abondante ? C'est là l'effet de la bonté de Bacchus. Refusent-ils l'une ou l'autre leurs présents? C'est au déplaisir de ces divinités offensées qu'il faut l'attribuer. L'arbre, qui fleurit aujourd'hui et demain dépérit, est habité par une Dryade, de la santé ou de la maladie de qui dépendent 
ses apparences diverses. La source, qui tantôt libère un flot copieux, et tantôt un maigre filet, qui parfois apparaît claire et limpide, et d'autres fois boueuse et troublée, est dans tous ses changements sous l'influence de la Naìade qui y demeure. De là l'origine du Polythéisme, et de cette superstition vulgaire qui attribue tous les événements irréguliers de la nature à la faveur ou au déplaisir d'êtres intelligents, quoique invisibles, aux dieux, démons, sorcières, génies, fées. Car on peut observer que, dans toutes les religions polythéistes, chez les sauvages, autant que dans les âges primitifs de l'antiquité païenne, ce ne sont que les événements irréguliers de la nature qui sont attribués à l'action et au pouvoir de leurs dieux. C'est par la nécessité de leur propre nature que le feu brûle, et que l'eau rafraîchit; que les corps lourds tombent, et que les substances plus légères s'envolent; et l'on ne redoutait jamais que la main invisible de Jupiter fût employée dans ces matières. Mais le tonnerre et l'éclair, les tempêtes et l'éclat du soleil, ces événements plus irréguliers, étaient attribués à sa faveur, ou à sa colère. L'homme, qui représentait le seul pouvoir doué d'intention qu'ils connaissaient, n'agit jamais que pour arrêter, ou modifier le cours, que les événements naturels, laissés à eux-mêmes, prendraient. Ces autres êtres intelligents, qu'ils imaginaient, mais qu'ils ne connaissaient pas, étaient naturellement supposés agir de la même façon ; non pour s'employer à soutenir le cours ordinaire des choses, qui allait de soi, mais pour l'arrêter, le contrarier, et le troubler. Et ainsi, dans les premiers âges du monde, la superstition la plus basse et la plus pusillanime suppléait la Philosophie " (HA, III, 2, p. 48-50). 